\title{
MRI in Pituitary Iron Overload: Current Perspective and Future Directions
}

\author{
Deeksha Bhalla ${ }^{1} \cdot$ Manisha Jana ${ }^{1}$ (D) \\ Received: 6 May 2021 / Accepted: 12 May 2021 / Published online: 22 May 2021 \\ (C) Dr. K C Chaudhuri Foundation 2021
}

Magnetic resonance imaging (MRI) and serum ferritin have emerged as the dominant noninvasive markers for estimation of iron overload in patients receiving chronic blood transfusions for hemolytic anemia. The values of $\mathrm{T} 2, \mathrm{~T} 2 *$ and rate constants derived from them (R2 and $\mathrm{R} 2 *$, respectively) are calculated, based on the physical principle of inhomogeneity in magnetic field produced by susceptibility effects of iron in body tissues. Liver iron concentration (LIC) estimates from R2 and R2* have been validated early on and are in routine clinical use [1]. Similarly, left ventricular function has been shown to correlate with cardiac T2* measurements [2], which is used as a biomarker for preclinical iron accumulation.

The endocrine effects of iron excess have thus far been studied extensively in the pancreas. Pancreatic iron overload serves as a marker for excess circulating non-transferrin-bound iron (NTBI); and not only relates to glucose dysfunction, but is also an indicator of imminent cardiac dysfunction and hypogonadotropic hypogonadism. A pancreatic R2* threshold of $100 \mathrm{~Hz}$ has been suggested for risk stratification [3].

Both short stature (SS) and hypogonadism in thalassemia are the result of a complex interplay of multiple factors including direct gonadal toxicity of iron and chronic anemia among others. Pituitary iron overload leading to endocrine failure is yet another cause. The article by Nayak and colleagues [4] discusses this relatively less explored domain. They estimated pituitary volume (PV) and iron concentration on 3T MRI in 57 cases and 30 controls. The authors derived the correlation of pituitary $\mathrm{T} 2 *, \mathrm{R} 2$, and volume with other surrogates of iron overload, and found that pituitary R2, like pancreatic R2 has a significant negative correlation with cardiac T2*. Patients with hypogonadism had significantly lower PV (mean $180.27 \mathrm{~mm}^{3}$ versus $\left.285.04 \mathrm{~mm}^{3}\right)$ and $\mathrm{T} 2 *$ value $(8.18 \mathrm{~ms}$

Manisha Jana

manishajana@gmail.com

1 Department of Radiodiagnosis, All India Institute of Medical Sciences, New Delhi 110029 India versus $16.48 \mathrm{~ms}$ ) compared to controls; the former was also observed in those with SS (mean $231.59 \mathrm{~mm}^{3}$ versus $307.36 \mathrm{~mm}^{3}$ ). However, since reduced PV and iron overload are not the only reasons for SS in these children, hormone assay is essential to establish causality.

It would have been useful if the authors had established age- and gender-based nomograms for PV and R2 using a larger number of controls for the Indian population. Such reference data would enable estimation of $\mathrm{z}$ score, as used in prior studies [5], which is a more meaningful predictor of outcome than a single value cutoff.

The authors have demonstrated the feasibility of using a 3T MRI system; however, any advantages or limitations over $1.5 \mathrm{~T}$ systems have not been analyzed. This is particularly important as air in sphenoid sinus and pituitary iron may create extensive artifacts due to susceptibility effect (higher in 3T systems) and hamper adequate planimetry for volume calculation.

Thus, this study highlights an important concern of pituitary iron overload causing growth deficiency and pubertal defect, which must be considered while planning iron chelation therapy. However, prior to using pituitary R2 or volume as biomarkers for therapy, larger trials with objective assessment of benefit analysis need to be performed to determine risk thresholds.

\section{Declarations}

Conflict of Interest None.

\section{References}

1. Wood JC. Estimating tissue iron burden: current status and future prospects. Br J Haematol. 2015;170:15-28.

2. Anderson LJ, Holden S, Davis B, et al. Cardiovascular T2-star (T2*) magnetic resonance for the early diagnosis of myocardial iron overload. Eur Heart J. 2001;22:2171-9. 
3. Noetzli LJ, Mittelman SD, Watanabe RM, Coates TD, Wood JC. Pancreatic iron and glucose dysregulation in thalassemia major. Am J Hematol. 2012;87:155-60.

4. Nayak AM, Choudhari A, Patkar DP, Merchant RH. Pituitary volume and iron overload evaluation by $3 \mathrm{~T} \mathrm{MRI}$ in Thalassemia. Indian J Pediatr. 2021. https://doi.org/10.1007/s12098-020-03629-w.
5. Noetzli LJ, Panigrahy A, Mittelman SD, et al. Pituitary iron and volume predict hypogonadism in transfusional iron overload. Am J Hematol. 2012;87:167-71.

Publisher's Note Springer Nature remains neutral with regard to jurisdictional claims in published maps and institutional affiliations. 